\title{
Transferability of Skills across Sectors and Heterogeneous Displacement Costs - Online Appendix
}

\author{
Moises Yi Steffen Mueller Jens Stegmaier
}

\section{Selection Model}

The full selection model is given below. Given that we only focus on one origin sector (manufacturing), we omit the $o$ subscripts for simplicity. The number of potential destination sectors is 9 .

$$
\begin{aligned}
\Delta \ln \text { wage }_{i d} & =X_{i}^{\prime} \beta_{d}+\Delta K_{i}^{\prime} \gamma+\epsilon_{i d} \\
V_{i d} & =X_{i}^{\prime} \tilde{\beta}_{d}+\Delta K_{i}^{\prime} \tilde{\gamma}+\sum_{s=1}^{9} \gamma_{d}^{s} C N_{i s}+\eta_{i d} \quad(d=1, \ldots, 9) \\
D_{i d} & = \begin{cases}1 & \Longleftrightarrow V_{i d}=\max \left(V_{i 1}, \ldots, V_{i 9}\right) \\
0 & \text { otherwise }\end{cases}
\end{aligned}
$$

$\Delta \ln w_{a g e}$ represents the log change in wages for worker $i$ moving from manufacturing to destination sector $d$. $V_{i d}$ represents the utility worker $i$ obtains in sector $d$, which is determined by pecuniary and non-pecuniary factors. The latter include our coworker selection instruments $C N_{i s}$ (the number of worker $i$ 's past coworkers present in sector $s$ at the time of worker $i$ 's displacement). Workers sort into the sector that maximizes their utility $V_{i d}$.

We assume the error terms in the selection equation $\left(\eta_{i d}\right)$ are i.i.d extreme value type I. Furthermore, we impose the following linearity assumption on the structure of the error terms:

$$
E\left[\epsilon_{i d} \mid \eta_{i 1} \ldots \eta_{i 9}\right]=\sigma \frac{\sqrt{6}}{\pi} \sum_{s} r_{d}^{s}\left(\eta_{i s}-E\left[\eta_{i s}\right]\right)
$$

These assumptions are based on work by Dubin and McFadden (1984) and described in detail in Bourguignon et al. (2007), and allow us to write the outcome equation in the following manner:

$$
\Delta \ln \text { wage }_{i d}=X_{i}^{\prime} \beta_{d}+\Delta K_{i}^{\prime} \gamma+\lambda_{d}\left(p_{i 1} \ldots, p_{i 9}\right)+\omega_{i d}
$$

where $\lambda_{d}(\bullet)$ represents the selection correction function with the probabilities $p_{i s}$ 's (that indi- 
vidual $i$ from moves to sector $s$ ) as arguments. Dubin and McFadden (1984) show that under our assumptions this correction function takes the form:

$$
\lambda_{d}(\bullet)=\sigma \frac{\sqrt{6}}{\pi}\left[\sum_{s \neq d} r_{d}^{s}\left(\frac{p_{i s} \ln p_{i s}}{1-p_{i s}}\right)-r_{d}^{d} \ln p_{i d}\right]
$$

\section{List of Sectors and Sample Descriptives}

Table A.1: List of Sectors

\begin{tabular}{ll}
\hline \hline$\#$ & Sector \\
\hline 1 & Agriculture, mining, utilities \\
2 & Manufacturing \\
3 & Construction \\
4 & Retail \\
5 & Transportation \\
6 & Hotel, restaurants, low skill svcs \\
7 & Communication, finance, prof svcs \\
8 & Office and business support svcs \\
9 & Education, hospitals, personal svcs \\
\hline
\end{tabular}

Table A.2: Sample Descriptives (by destination sector)

\begin{tabular}{lccccc}
\hline \hline Industry & Age & Experience & $\triangle \log ($ wage $)$ & Coworker Network Size & $\mathrm{N}$ \\
\hline Agriculture, mining, utilities & 27.1 & 8.6 & 0.08 & 55.8 & 1,207 \\
Manufacturing & 27.9 & 9.9 & 0.09 & 42.7 & 115,713 \\
Construction & 25.8 & 7.6 & 0.14 & 30.3 & 7,651 \\
Retail & 27.3 & 8.8 & 0.10 & 54.2 & 13,169 \\
Transportation & 26.7 & 8.3 & 0.07 & 75.5 & 2,059 \\
Hotel, restaurants, low skill svcs & 23.7 & 5.6 & 0.03 & 13.6 & 966 \\
Communication, finance, prof svcs & 29.2 & 10.4 & 0.04 & 69.7 & 5,076 \\
Office and business support svcs & 28.6 & 9.9 & -0.04 & 71.4 & 4,410 \\
Education, hospitals, personal svcs & 29.7 & 11.3 & -0.12 & 46.9 & 2,750 \\
Total & 27.8 & 9.6 & 0.08 & 153,001 \\
\hline
\end{tabular}

Notes: Experience measured in years. Coworker network size includes coworkers in all potential destination sectors (after applying the restrictions described in Section I)

\section{First Stage Results}

The first stage estimates are presented in Table A.2. Each column represents a potential destination sector $d$. Each cell contains the estimated coefficient $\gamma_{d}^{s}$ for $C N_{i s}$ (the number of coworkers 
with jobs in sector $s$ at the time of worker i's switch). Hence, the entries on the diagonal indicate that having coworkers in sector $d$ increases the probability that worker $i$ moves to $d$. 
Table A.3: Multinomial Logit Model

\begin{tabular}{|c|c|c|c|c|c|c|c|c|c|}
\hline & 1 & 2 & 3 & 4 & 5 & 6 & 7 & 8 & 9 \\
\hline$C N_{i 1}$ & $\begin{array}{r}0.00819^{* * *} \\
(0.00183)\end{array}$ & $\begin{array}{r}0 \\
(.)\end{array}$ & $\begin{array}{r}0.00156 \\
(0.00363)\end{array}$ & $\begin{array}{r}-0.00174 \\
(0.00353)\end{array}$ & $\begin{array}{l}0.00522^{* *} \\
(0.00196)\end{array}$ & $\begin{array}{l}-0.00933 \\
(0.0386)\end{array}$ & $\begin{array}{r}-0.00437 \\
(0.00272)\end{array}$ & $\begin{array}{c}0.00368^{*} \\
(0.00179)\end{array}$ & $\begin{array}{r}-0.00650 \\
(0.00555)\end{array}$ \\
\hline$C N_{i 2}$ & $\begin{array}{r}-0.000261 \\
(0.000341)\end{array}$ & $\begin{array}{r}0 \\
(.)\end{array}$ & $\begin{array}{c}-0.000853^{*} \\
(0.000357)\end{array}$ & $\begin{array}{r}-0.000528^{* *} \\
(0.000161)\end{array}$ & $\begin{array}{r}0.000312^{*} \\
(0.000128)\end{array}$ & $\begin{array}{r}-0.00169 \\
(0.00317)\end{array}$ & $\begin{array}{c}-0.000105 \\
(0.000109)\end{array}$ & $\begin{array}{r}-0.000154 \\
(0.000145)\end{array}$ & $\begin{array}{r}-0.000200 \\
(0.000156)\end{array}$ \\
\hline$C N_{i 3}$ & $\begin{array}{r}0.00123 \\
(0.00385)\end{array}$ & $\begin{array}{r}0 \\
(.)\end{array}$ & $\begin{array}{l}0.0121^{* * *} \\
(0.00238)\end{array}$ & $\begin{array}{c}0.00590^{* *} \\
(0.00204)\end{array}$ & $\begin{array}{r}0.00355 \\
(0.00207)\end{array}$ & $\begin{array}{r}-0.00840 \\
(0.0228)\end{array}$ & $\begin{array}{r}0.00387 \\
(0.00212)\end{array}$ & $\begin{array}{r}-0.00248 \\
(0.00274)\end{array}$ & $\begin{array}{r}-0.00226 \\
(0.00306)\end{array}$ \\
\hline$C N_{i 4}$ & $\begin{array}{r}-0.00113 \\
(0.00143)\end{array}$ & $\begin{array}{l}0 \\
(.)\end{array}$ & $\begin{array}{r}-0.00131 \\
(0.00169)\end{array}$ & $\begin{array}{c}0.00383^{\text {***}} \\
(0.000439)\end{array}$ & $\begin{array}{r}0.00174^{*} \\
(0.000840)\end{array}$ & $\begin{array}{r}-0.00751 \\
(0.00732)\end{array}$ & $\begin{array}{r}0.000354 \\
(0.000525)\end{array}$ & $\begin{array}{l}0.00310^{* * *} \\
(0.000657)\end{array}$ & $\begin{array}{c}0.00284^{* * *} \\
(0.000626)\end{array}$ \\
\hline$C N_{i 5}$ & $\begin{array}{r}0.000537 \\
(0.00137)\end{array}$ & $\begin{array}{l}0 \\
(.)\end{array}$ & $\begin{array}{r}0.000633 \\
(0.00255)\end{array}$ & $\begin{array}{r}0.000376 \\
(0.00232)\end{array}$ & $\begin{array}{r}0.00118^{*} \\
(0.000469)\end{array}$ & $\begin{array}{r}0.00260 \\
(0.00239)\end{array}$ & $\begin{array}{r}0.000368 \\
(0.000590)\end{array}$ & $\begin{array}{c}0.0000765 \\
(0.000914)\end{array}$ & $\begin{array}{r}0.000550 \\
(0.000637)\end{array}$ \\
\hline$C N_{i 6}$ & $\begin{array}{r}0.00697 \\
(0.0134)\end{array}$ & $\begin{array}{l}0 \\
(.)\end{array}$ & $\begin{array}{r}0.0115 \\
(0.00843)\end{array}$ & $\begin{array}{r}-0.00369 \\
(0.00527)\end{array}$ & $\begin{array}{r}-0.00668 \\
(0.00620)\end{array}$ & $\begin{array}{c}0.0549^{* *} \\
(0.0178)\end{array}$ & $\begin{array}{r}-0.00368 \\
(0.00434)\end{array}$ & $\begin{array}{c}-0.00804 \\
(0.00631)\end{array}$ & $\begin{array}{c}-0.0147^{*} \\
(0.00643)\end{array}$ \\
\hline$C N_{i 7}$ & $\begin{array}{r}-0.000292 \\
(0.000291)\end{array}$ & $\begin{array}{l}0 \\
(.)\end{array}$ & $\begin{array}{c}0.000751^{* *} \\
(0.000247)\end{array}$ & $\begin{array}{r}0.0000138 \\
(0.000217)\end{array}$ & $\begin{array}{c}-0.00281^{* * *} \\
(0.000852)\end{array}$ & $\begin{array}{r}0.00165^{*} \\
(0.000696)\end{array}$ & $\begin{array}{c}0.00321^{*} \\
(0.00138)\end{array}$ & $\begin{array}{r}-0.000861^{* *} \\
(0.000308)\end{array}$ & $\begin{array}{r}-0.000614 \\
(0.000517)\end{array}$ \\
\hline$C N_{i 8}$ & $\begin{array}{r}-0.0125 \\
(0.00952)\end{array}$ & $\begin{array}{r}0 \\
(.)\end{array}$ & $\begin{array}{r}-0.0162 \\
(0.0117)\end{array}$ & $\begin{array}{r}0.00241 \\
(0.00144)\end{array}$ & $\begin{array}{r}0.00717^{* * *} \\
(0.00204)\end{array}$ & $\begin{array}{r}0.0108 \\
(0.00616)\end{array}$ & $\begin{array}{r}0.000169 \\
(0.000111)\end{array}$ & $\begin{array}{r}0.00814^{* * *} \\
(0.00218)\end{array}$ & $\begin{array}{r}-0.0000996 \\
(0.00272)\end{array}$ \\
\hline$C N_{i 9}$ & $\begin{array}{l}0.0112^{* * *} \\
(0.00216)\end{array}$ & $\begin{array}{r}0 \\
(.)\end{array}$ & $\begin{array}{r}-0.00319 \\
(0.00349)\end{array}$ & $\begin{array}{c}-0.00567^{*} \\
(0.00261)\end{array}$ & $\begin{array}{r}0.00175 \\
(0.00331)\end{array}$ & $\begin{array}{l}-0.0700^{*} \\
(0.0300)\end{array}$ & $\begin{array}{c}0.00471^{* *} \\
(0.00159)\end{array}$ & $\begin{array}{r}0.000927 \\
(0.00208)\end{array}$ & $\begin{array}{l}0.0124^{* * *} \\
(0.00281)\end{array}$ \\
\hline$\chi^{2}$ & $\begin{array}{r}69.429 \\
{[0.0000]}\end{array}$ & & $\begin{array}{r}40.128 \\
{[0.0000]}\end{array}$ & $\begin{array}{l}132.077 \\
{[0.0000]}\end{array}$ & $\begin{array}{l}105.846 \\
{[0.0000]}\end{array}$ & $\begin{array}{r}55.402 \\
{[0.0000]}\end{array}$ & $\begin{array}{l}127.160 \\
{[0.0000]}\end{array}$ & $\begin{array}{l}120.516 \\
{[0.0000]}\end{array}$ & $\begin{array}{l}105.802 \\
{[0.0000]}\end{array}$ \\
\hline $\mathrm{N}$ & & & & & 153001 & & & & \\
\hline
\end{tabular}

Notes: First-stage estimates for coworker network regressors. Each column represents a potential destination sector $d$. Each cell contains the estimated coefficient $\gamma_{d}^{s}$ for $C N_{i s}$ (the number of coworkers with jobs in sector $s$ at the time of worker i's switch). $\chi^{2}$ statistics test for joint significance of coefficients $\gamma_{d}^{s}$ (for $s=1, \ldots, 9$ ). Robust standard errors in parenthesis, p-values in brackets. Base outcome=manufacturing. Names of sectors listed on Table A.1. 


\section{References}

Bourguignon, F., Fournier, M., and Gurgand, M. (2007). Selection Bias Corrections Based On The Multinomial Logit Model: Monte Carlo Comparisons. Journal of Economic Surveys, 21(1):174-205.

Dubin, J. A. and McFadden, D. L. (1984). An Econometric Analysis of Residential Electric Appliance Holdings and Consumption. Econometrica, 52(2):345-62. 